\title{
Low-Temperature Synthesis and Thermodynamic and Electrical Properties of Barium Titanate Nanorods
}

\author{
Florentina Maxim, ${ }^{1}$ Daniela Berger, ${ }^{2}$ Florina Teodorescu, ${ }^{1}$ Cristian Hornoiu, ${ }^{1}$ \\ Cecilia Lete, ${ }^{1}$ and Speranta Tanasescu ${ }^{1}$ \\ 1 "Ilie Murgulescu" Institute of Physical Chemistry, Splaiul Independentei No. 202, 060021 Bucharest, Romania
2Department of Inorganic Chemistry, Physical Chemistry and Electrochemistry, University "Politehnica" of Bucharest,
Polizu Street No. 1-7, 011061 Bucharest, Romania
}

Correspondence should be addressed to Florentina Maxim; fmaxim@icf.ro and Daniela Berger; danaberger01@yahoo.com

Received 12 January 2015; Revised 20 March 2015; Accepted 24 March 2015

Academic Editor: Joydeep Dutta

Copyright (C) 2015 Florentina Maxim et al. This is an open access article distributed under the Creative Commons Attribution License, which permits unrestricted use, distribution, and reproduction in any medium, provided the original work is properly cited.

Studies regarding the morphology dependence of the perovskite-type oxides functional materials properties are of recent interest. With this aim, nanorods (NRs) and nanocubes (NCs) of barium titanate $\left(\mathrm{BaTiO}_{3}\right)$ have been successfully synthesized via a hydrothermal route at temperature as low as $408 \mathrm{~K}$, employing barium acetate, titanium isopropoxide, and sodium hydroxide as reagents without any surfactant or template. Scanning electron microscopy (SEM), transmission electron microscopy (TEM), and X-ray powder diffraction (XRD), used for the morphology and structure analyses, showed that the NRs were formed by an oriented attachment of the NCs building-blocks with $20 \mathrm{~nm}$ average crystallites size. The thermodynamic properties represented by the relative partial molar free energies, enthalpies, and entropies of the oxygen dissolution in the perovskite phase, as well as the equilibrium partial pressure of oxygen, indicated that NRs powders have lower oxygen vacancies concentration than the NCs. This NRs characteristic, together with higher tetragonallity of the structure, leads to the enhancement of the dielectric properties of $\mathrm{BaTiO}_{3}$ ceramics. The results presented in this work show indubitably the importance of the nanopowders morphology on the material properties.

\section{Introduction}

Since its discovery during the Second World War, barium titanate $\left(\mathrm{BaTiO}_{3}\right)$ has been extensively studied because of its excellent dielectric, ferroelectric, and piezoelectric properties providing new opportunities for a wide range of applications directed to the electronic devices industry $[1,2]$. A renewed scientific interest, especially for nanostructured $\mathrm{BaTiO}_{3}$, has been shown because this is a lead-free ferroelectric material that can overcome the environmental problems related to the release of lead during the production process of lead-based materials [3]. However, when considering the nanostructured forms of barium titanate it must be taken into account that, at nanoscale, a pseudo cubic, nonferroelectric structure, with submicrometric particle size and with a low dielectric constant value, is stabilized at room temperature [4]. Among barium titanate nanostructures, the one-dimensional (1D) morphology as nanotubes, nanorods, or nanowires presents a special interest because this kind of nanoparticles can conserve its high dielectric constant and ferroelectric switchability at nanoscale [5]. Thereby, extensive research has been conducted on different synthesis approaches to obtain 1D barium titanate nanostructures and the most reported are the template assisted sol-gel $[6,7]$, electrophoretic $[8,9]$, and hydrothermal [10-12] methods. However, when choosing a synthesis method for $1 \mathrm{D}$ perovskite-type oxides it must be considered that this is capable of ensuring the complete control over the structure, size, and morphology for the enhanced functional properties at nanoscale preservation. In this view, the hydrothermal method is a very good approach, as it has been demonstrated that the structure, size, and shapecontrolled $\mathrm{BaTiO}_{3}$ crystalline nanoparticles can be obtained by hydrothermal treatments of precursors at relatively low temperatures $(<573 \mathrm{~K})$ [13-16]. 
The low temperature and no need of sophisticated equipment make from the hydrothermal method a cost and time effective synthesis procedure. However, for $1 \mathrm{D} \mathrm{BaTiO}$ nanostructures, the template assisted methods reported in the literature have the drawback of two-step procedure as firstly the titanium precursor is obtained in 1D morphology, and then the reaction parameters with the barium source must be carefully chosen to enable shape retention during the hydrothermal treatment [10-12]. Therefore, to keep the cost and the duration for the hydrothermal procedure reduced, one-step approaches to achieve $1 \mathrm{D}$ growth should be evaluated. Here we propose one-step hydrothermal synthesis procedure for the formation of $1 \mathrm{D}$ barium titanate by self-assembly nanostructured building-blocks [17]. The success of this strategy under hydrothermal conditions has been demonstrated for the preparation of perovskite $\mathrm{KNbO}_{3}$ nanowires $[18,19]$. The authors showed that highly anisotropic potassium niobate $1 \mathrm{D}$ nanostructures grown from isotropic hydrothermal medium have slightly distorted cubes as building blocks [19]. Maxim et al. demonstrated that $\mathrm{BaTiO}_{3}$ nanocubes with various sizes can be obtained by a sol-gel hydrothermal method [20].

To the best of our knowledge there are no studies reporting the hydrothermal synthesis of $1 \mathrm{D}$ barium titanate by nanocubes self-assembly strategy. This paper demonstrated that $\mathrm{BaTiO}_{3}$ nanorods can be obtained by the mentioned approach. For a more complex characterization, the morphological and structural analysis has been coupled with the evaluation of the thermodynamic and electrical characteristics in relation to significant changes in the overall concentration of defects. By the comparative study of nanorods and nanocubes structures, new features related to the modifications in properties connected with the nanocrystalline state were evidenced.

\section{Materials and Methods}

The barium titanate synthesis was performed in a high pressure Berghof BR-100 reactor. The precursors and the basic synthesis procedure were previously reported by Maxim et al. [20]. In the present study the synthesis parameters were optimized in order to obtain $1 \mathrm{D}$ nanostructures. The sol containing the barium and titanium ions was prepared by adding drop wise $5 \mathrm{~mL} \mathrm{Ti}\left(\mathrm{C}_{3} \mathrm{H}_{7} \mathrm{O}\right)_{4}$ (Sigma Aldrich > 97\%), under vigorous stirring, in a solution of $\mathrm{Ba}\left(\mathrm{CH}_{3} \mathrm{COO}\right)_{2}$ (Merck 99.0-102.0\%) in glacial acetic acid (Sigma Aldrich). The Ba/Ti molar ratio was 1 . The sol was precipitated in aqueous solution of sodium hydroxide (Merck > 99\%). The quantity of the acetic acid was calculated after the neutralization with the hydroxide to give a $\mathrm{pH}$ higher than 13 . The reaction mixture was transferred into the reactor and hydrothermally treated at $408 \mathrm{~K}\left(135^{\circ} \mathrm{C}\right)$ for $24 \mathrm{~h}$. One synthesis under magnetic stirring and another without magnetic stirring have been performed. Then, the resulted powders were filtered off and washed with distilled water until neutral $\mathrm{pH}$. The as prepared powders were dried in air at $60^{\circ} \mathrm{C}$ for $12 \mathrm{~h}$.

The powders morphology was analyzed by scanning electron microscopy (SEM) with a FEI Quanta 3D FEG microscope operated at up to $10 \mathrm{kV}$ acceleration voltages and by transmission electron microscopy (TEM) with a FEI Tecnai G2-F30 instrument at $300 \mathrm{kV}$. Structural analysis was conducted by X-ray diffraction (XRD) technique at room temperature, using a Rigaku MiniFlex II diffractometer with $\mathrm{Cu} \mathrm{K} \alpha$ radiation $(\lambda=0.15406 \mathrm{~nm})$, in $20^{\circ}-80^{\circ} 2 \theta$ range, with a step length of $0.02^{\circ}$ and a fixed counting time $=1 \mathrm{~s} /$ step.

A solid state electrochemical method was used to obtain the thermodynamic data represented by the relative partial molar free energies, enthalpies, and entropies of oxygen dissolution in the perovskite phase $\left(\Delta \bar{G}_{\mathrm{O}_{2}}, \Delta \bar{H}_{\mathrm{O}_{2}}\right.$, and $\left.\Delta \bar{S}_{\mathrm{O}_{2}}\right)$, as well as the equilibrium partial pressures of oxygen as a function of temperature. An electrochemical cell containing 12.84 wt.\% yttria stabilized zirconia solid electrolyte and an iron-wüstite reference electrode has been used. The design of the setup, as well as the theoretical and experimental considerations related to the applied method, has been previously described [21]. For these measurements the hydrothermal powders were pressed into pellets weighting $\sim 0.1 \mathrm{~g}$. Measurements were performed in vacuum at a residual gas pressure of $10^{-7}$ atm. The electromotive force (EMF) was measured with a Keithley 2000 Multimeter, at $50 \mathrm{~K}$ intervals between $773 \mathrm{~K}$ and $1273 \mathrm{~K}$, each time waiting until equilibrium conditions were obtained. Equilibrium conditions were achieved when the EMF values for increasing and decreasing temperatures agreed within $\pm 1 \mathrm{mV}$ during $10 \mathrm{~min}$. The free energy change of the galvanic cell is given by the expression

$$
\Delta G_{\text {cell }}=\mu_{\mathrm{O}_{2}}-\mu_{\mathrm{O}_{2}}(\text { ref })=4 F E
$$

where $E$ is the steady-state EMF of the cell in volts, $\mu_{\mathrm{O}_{2}}$ and $\mu_{\mathrm{O}_{2}}$ (ref) are the oxygen chemical potential of the sample and the reference electrode, respectively, and $F$ is the Faraday constant. Knowing the experimental values of the EMF, the relative partial molar free energy of the solution of oxygen in the perovskite phase and hence the pressures of oxygen in equilibrium with the solid can be calculated based on the following formula [21]:

$$
\Delta \bar{G}_{\mathrm{O}_{2}}=R T \log p_{\mathrm{O}_{2}}
$$

The relative partial molar enthalpies and entropies were obtained according to the known relationships [21]:

$$
\begin{gathered}
\frac{\partial\left(\Delta \bar{G}_{\mathrm{O}_{2}} / T\right)}{\partial T}=-\frac{\Delta \bar{H}_{\mathrm{O}_{2}}}{T^{2}} \\
\Delta \bar{G}_{\mathrm{O}_{2}}=\Delta \bar{H}_{\mathrm{O}_{2}}-T \Delta \bar{S}_{\mathrm{O}_{2}} .
\end{gathered}
$$

For the dielectric properties measurements the hydrothermal powders were pressed into pellets of $13 \mathrm{~mm}$ in diameter and around $1.3 \mathrm{~mm}$ thickness. For sintering, the pellets were thermally treated at $1623 \mathrm{~K}$ for $2 \mathrm{~h}$ in a Carbolite furnace with a heating/cooling rate of $2 \mathrm{~K} / \mathrm{min}$. Silver electrodes were painted onto both surfaces. A frequency response analyzer, Solartron Analytical 1255A, was used to obtain the dielectric constant (permittivity) and the dielectric losses data from room temperature to about $493 \mathrm{~K}$ in the $100 \mathrm{~Hz}$ to $1 \mathrm{MHz}$ frequency range. 




(a)



(c)

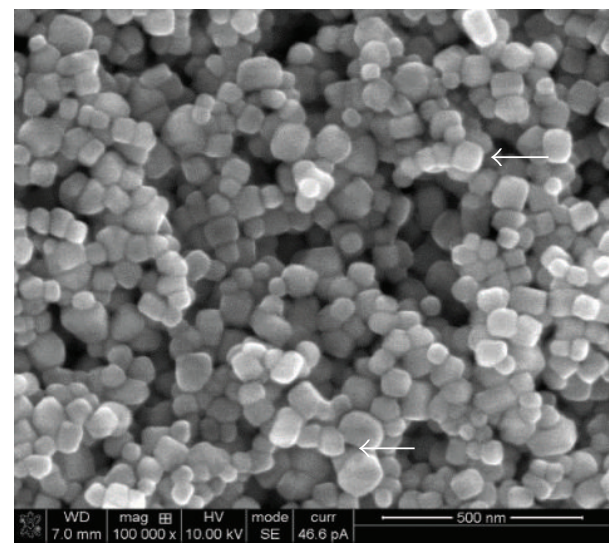

(b)



(d)

Figure 1: SEM images ((a), (c)) and higher magnification (>100 000x) SEM micrographs ((b), (d)) of the samples obtained by hydrothermal treatment with stirring ((a) and (b), BTNCs) and without stirring ((c) and (d), BTNRs).

\section{Results and Discussion}

SEM investigation showed that cube-shaped nanoparticles with narrow size distribution and average dimension of $55 \mathrm{~nm}$ were formed after the hydrothermal treatment with stirring (Figures 1(a) and 1(b)), SEM investigation of the sample denoted BTNCs showed that the SEM analysis of the material prepared without stirring (Figures 1(c) and $1(\mathrm{~d})$ ) revealed the formation of faceted elongated particles with average diameter of $147 \mathrm{~nm}$ and aspect ratio around 3 suggesting the one-dimensional feature of the sample labelled BTNRs. The value of the aspect ratio indicates the formation of nanorods [22]. As can be observed in the high magnification SEM micrographs (Figures 1(b) and 1(d)) the nanocubes have the tendency to aggregate in one direction suggesting the self-organization of these nanostructures (arrows in Figure 1(b)). Indeed, the nanorods seem to be formed by oriented aggregation of faceted cuboids particles (Figure 1(d)). These observations indicate the possibility of barium titanate particles growth by an oriented attachment mechanism $[23,24]$ involving nanoparticles with common crystallographic orientations which join together by a planar interface to form a larger particle. To give further insight on the growth mechanism of the nanorods, TEM investigation was used (Figure 2). The bright field image (Figure 2(a)) shows a barium titanate nanorod with smooth edges and parallelepiped shape. The dark field image (Figure 2(b)) shows the single crystal feature as the brightness/contrast is uniform along the entire 1D particle. Additionally, in the dark field image one can observe the planar crystal defects which are marked with arrows in Figure 2(b). These planar defects can be attributed to dislocations generated by imperfect oriented aggregation of the building block nanocrystals [25]. The selected area electron diffraction (SAED, square mark in Figure 2(a)) presented in Figure 2(c) indicates once again that the nanorods have a single crystal characteristics and the growth axis of the nanorods is along (001) crystallographic axis of $\mathrm{BaTiO}_{3}$, which is the polar axis of the tetragonal structure. All TEM results are in accordance with the SEM analysis (Figure $1(\mathrm{~d})$ ) and force the idea that the BTNRs could be formed under hydrothermal conditions by a selfassembly oriented attachment mechanism. However, a more 


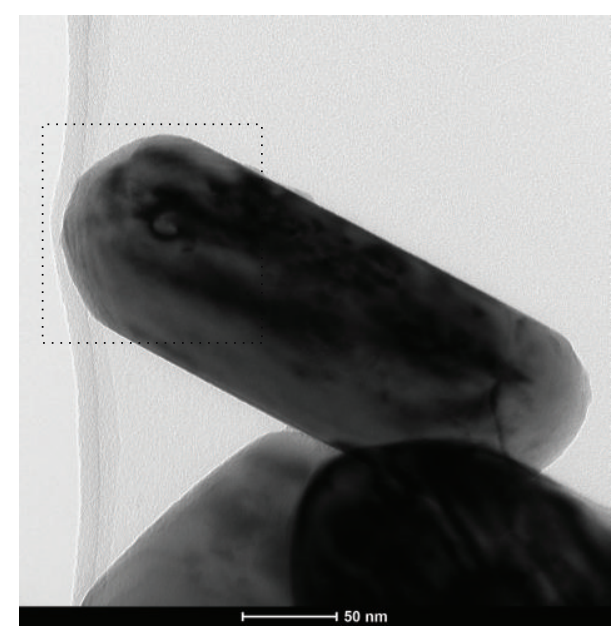

(a)

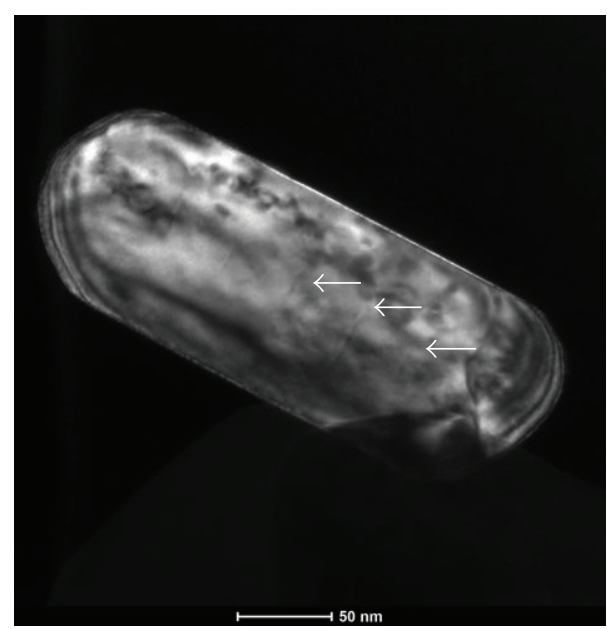

(b)

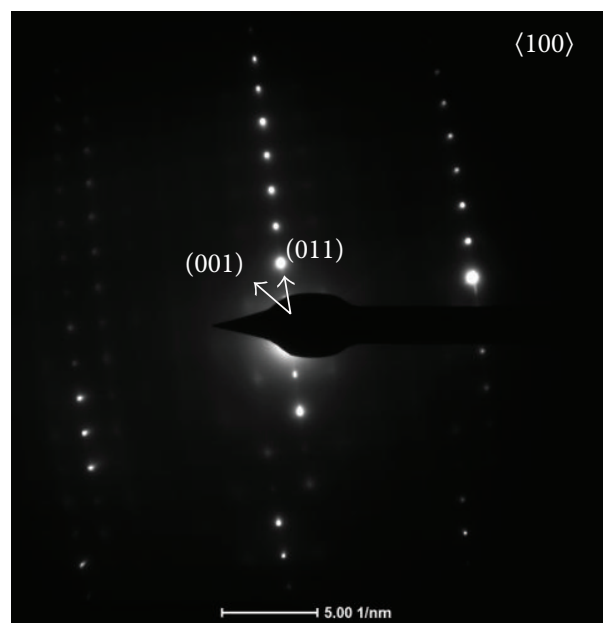

(c)

FIGURE 2: TEM analysis of BTNRs sample in bright field (a), dark field (b), and selected area electron diffraction (SAED) of the square marked area $(\mathrm{c})$.

detailed growth kinetic study must be performed in order to discriminate between various possible mechanisms (Oswald ripening versus oriented attachment [24]).

Further clarifications regarding the structure of $\mathrm{BaTiO}_{3}$ nanopowder samples were obtained by XRD (Figure 3 ). It is found that the XRD patterns of both samples can be indexed with JCPDS 75-0211 suggesting the formation of $\mathrm{BaTiO}_{3}$ cubic perovskite phase, characteristic to the barium titanate powders obtained by wet chemical methods, at low temperatures [26-28]. Moreover, no secondary phase of barium carbonate is formed, suggesting the reliability of the synthesis procedure proposed in this study (Figure 3(a)). The crystallite size calculated by Williams-Hall method was found to be around $20 \mathrm{~nm}$ for both samples and with a lattice parameter of $0.401 \mathrm{~nm}$. In Figure 3(b) the region around the cubic (200) reflection has been selected, $2 \theta \sim 45^{\circ}$, showing the fine structural differences between the samples. First difference is the relative intensity of the corresponding peak which is $25.5 \%$ for BTNRs and $31.4 \%$ for BTNCs, respectively. Moreover, the $2 \theta \sim 45^{\circ}$ peak has a FWHM value of $0.547^{\circ}$ in the case of BTNRs sample, higher than the corresponding value of $0.475^{\circ}$ for the BTNCs sample. These differences denote the broadening of the (200) reflection (Figure 3(b)) for the nanorods and can suggest the formation of predominant tetragonal structure, characteristic to barium titanate powders obtained by high temperature solid-state reaction [29]. To give better inside into the crystal structure, Raman spectroscopy has been performed. Although both samples reveal $\mathrm{BaTiO}_{3}$ Raman bands at around $185 \mathrm{~cm}^{-1}$ (characteristic to hydrothermally prepared barium titanate powders [30]), at $\sim 250,520$, and $720 \mathrm{~cm}^{-1}$ there are some differences suggesting different local structure for BTNCs when compared to BTNRs (Figure 4). The main difference is the broad band at around $299 \mathrm{~cm}^{-1}$ for the BTNCs characteristic to predominant cubic $\mathrm{BaTiO}_{3}$ phase and the sharp peak at around $305 \mathrm{~cm}^{-1}$ for BTNRs sample characteristic to tetragonal barium titanate structure [31]. As the functional properties (high dielectric constant, ferroelectric switchability) are conserved at nanoscale only for the ferroelectric tetragonal $\mathrm{BaTiO}_{3}$, the synthesis methods that allow the preparation of 


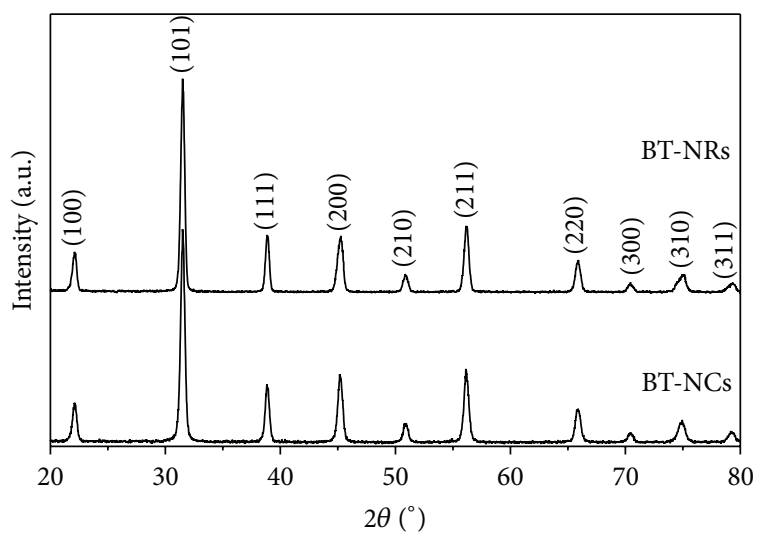

(a)

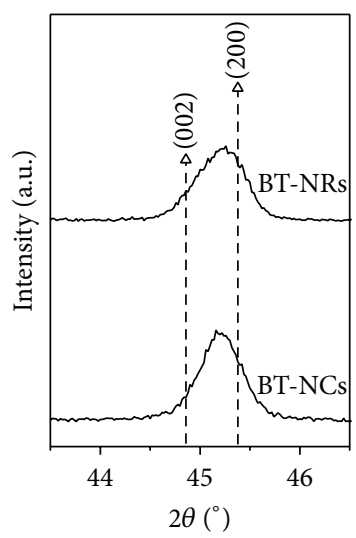

(b)

FIGURE 3: XRD patterns of BTNRs and BTNCs samples.

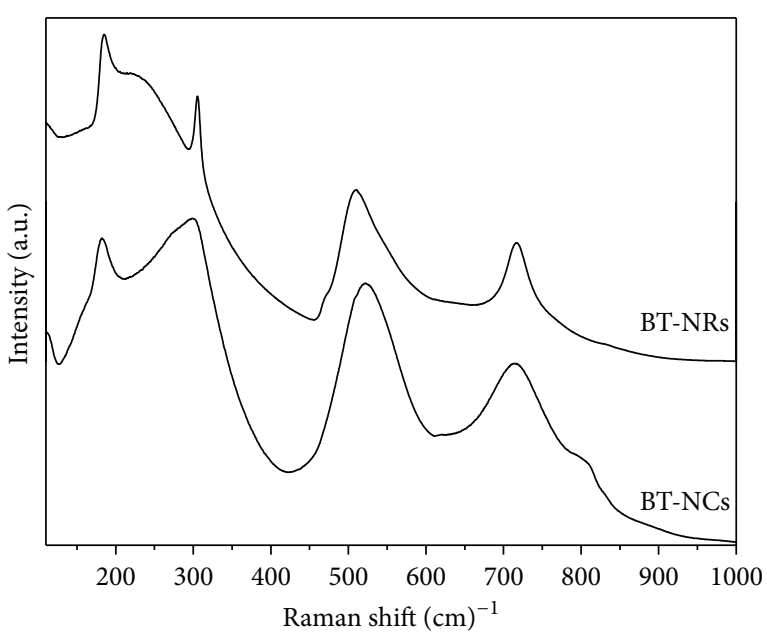

FIGURE 4: Raman spectra of BTNRs and BTNCs samples.

tetragonal barium titanate nanostructures at relatively low temperatures are highly desirable [32-36]. In the present study, based on the above results, it is demonstrated that the tetragonality of the nanorods is higher than in the case of barium titanate nanocubes obtained in the same conditions at $408 \mathrm{~K}$.

Moreover, in the Raman spectra (Figure 4) for the BTNCs sample it is observed that a shoulder at $\sim 807 \mathrm{~cm}^{-1}$ appears, indicating the lattice defect mode due to the deformation of lattice $\mathrm{OH}$ groups incorporated during the hydrothermal synthesis [30]. We have to note here that this Raman band is not present in the case of BTNRs sample. Thus, the structural differences between BTNRs and BTNCs samples can be discussed also in terms of lattice point defects, which are of special importance in functional materials as they control their electronic properties. For the hydrothermal $\mathrm{BaTiO}_{3}$, the hydroxyl groups incorporated in the perovskite lattice must be taken into consideration [37], which are responsible for the formation of donor-type oxygen vacancies and, according to the charge compensation, for the formation of acceptor-type barium and titanium vacancies [28]. Since the oxygen vacancies play a key role in aging and fatigue of ferroelectric materials by impeding domain wall motion or by acting as local disturbances of the polarization [38, 39], information about the concentration and mobility of this type of defects in barium titanate is of special interest. Some information about the correlation of the electrical and structural stability with the defect structure of $\mathrm{BaTiO}_{3}$ was obtained by conductivity and diffusivity measurements [40] or by theoretic calculations [41]. Tanasescu et al. demonstrated that information regarding the concentration and distribution of the oxygen vacancies into the perovskite lattice of complex oxide compounds can be obtained by determining the thermodynamic properties represented by the relative partial molar free energies, enthalpies, and entropies of the oxygen dissolution in the perovskite phase, as well as the equilibrium partial pressure of oxygen [21]. Here we present for the first time the comparison between the thermodynamic properties of hydrothermal prepared $\mathrm{BaTiO}_{3}$ with different morphologies. In Figure 5 the $\Delta \bar{G}_{\mathrm{O}_{2}}$ as a function of temperature, in the temperature range of $773-1273 \mathrm{~K}$, for BTNRs and BTNCs samples is presented. Three temperature regions can be observed for the variation of $\Delta \bar{G}_{\mathrm{O}_{2}}$. In the first region (temperature $<973 \mathrm{~K}$ ), one can observe that the $\Delta \bar{G}_{\mathrm{O}_{2}}$ values of BTNRs are with almost $100 \mathrm{~kJ} \mathrm{~mol}^{-1}$ lower than $\Delta \bar{G}_{\mathrm{O}_{2}}$ of BTNCs. The lower the partial molar free energy value is, the lower the oxygen vacancy concentration is [42]. In the $973-1173 \mathrm{~K}$ temperature range, the $\Delta \bar{G}_{\mathrm{O}_{2}}$ shows weak temperature dependence for nanorods and nanocubes morphologies. At temperatures higher than $1173 \mathrm{~K}$, the partial molar energy has an increasing trend for both samples. It is known that undoped barium titanate undergoes an $n$-type to $p$-type transition in its semiconducting behaviour at oxygen partial pressure higher than $10^{-6}$ atm $[43,44]$. In Figure 6 the variation of $\log p_{\mathrm{O}_{2}}$ as a function of $1 / T$ for the samples BTNCs and BTNRs is presented. First observation is that the $p_{\mathrm{O}_{2}}$ domain is below $10^{-10}$ atm, and then the data presented here are in the $n$-type regime. Second, it can be observed that the values of $p_{\mathrm{O}_{2}}$ obtained for the BTNRs sample are almost one order of magnitude lower than the corresponding values of the oxygen partial pressure of the BTNCs sample in 


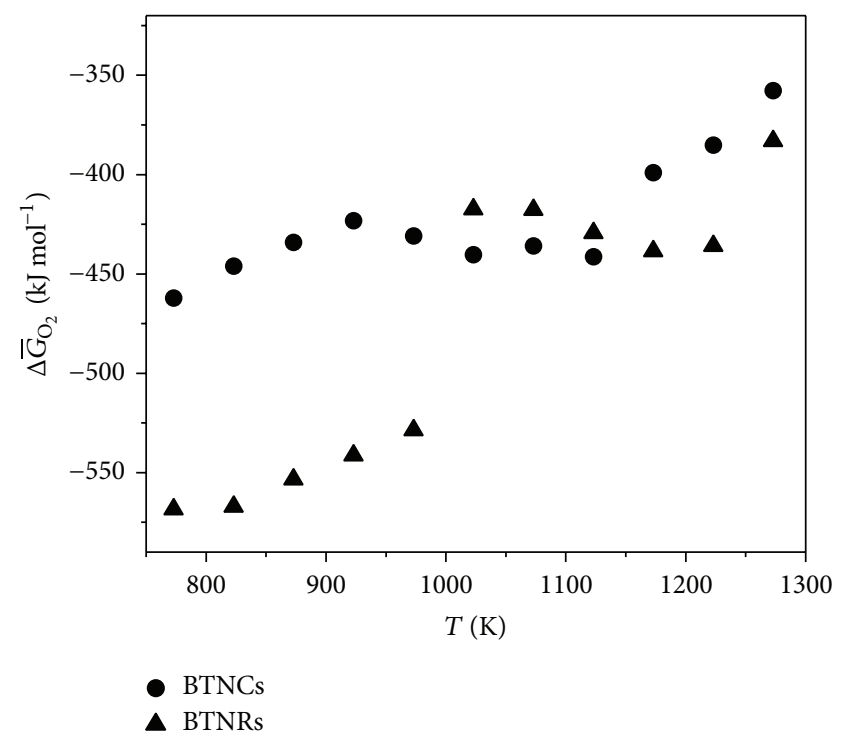

FIGURE 5: $\Delta \bar{G}_{\mathrm{O}_{2}}$ as a function of temperature for BTNRs and BTNCs samples.

the temperature range from 773 to $973 \mathrm{~K}$. Finally, at temperatures higher than $1073 \mathrm{~K}$, the $p_{\mathrm{O}_{2}}$ values obtained for both nanorods and nanocubes morphologies become comparable. Based on the above presented results, the variation of $\Delta \bar{G}_{\mathrm{O}_{2}}$ and $p_{\mathrm{O}_{2}}$ with temperature suggests the lowest concentration of oxygen vacancies in the perovskite lattice for the barium titanate with $1 \mathrm{D}$ morphology in the temperature range below $973 \mathrm{~K}$. Clarifications regarding the binding energy and the order in the oxygen sublattice of $\mathrm{BaTiO}_{3}$ perovskite structure can be achieved by determining the $\Delta \bar{H}_{\mathrm{O}_{2}}$ and $\Delta \bar{S}_{\mathrm{O}_{2}}$ values in temperature ranges in which the partial molar free energies are linear functions of temperature [21], namely, 773-873 K for BTNCs and 823-973 K for BTNRs. The $\Delta \bar{H}_{\mathrm{O}_{2}}$ values are $-677.62 \mathrm{~kJ} / \mathrm{mol}$ for BTNCs and $-776.42 \mathrm{~kJ} / \mathrm{mol}$ for BTNRs. The $\Delta \bar{S}_{\mathrm{O}_{2}}$ values are $-279.7 \mathrm{~J} / \mathrm{mol} \mathrm{K}$ for BTNCs and $-254.8 \mathrm{~J} / \mathrm{mol} \mathrm{K}$ for BTNRs. The decreasing of the enthalpy and the increasing value of the entropy for BTNRs suggest the increased binding energy of oxygen and the random distribution of the oxygen vacancies into the perovskite phase for the $1 \mathrm{D}$ barium titanate.

The electric properties of the dense ceramic bodies (SEM microstructure shown in Figure 7) obtained from the hydrothermal BTNCs and BTNRs powders have been evaluated and the variation of the permittivity and the dielectric losses with the frequency (from $1 \mathrm{~Hz}$ to $1 \mathrm{MHz}$ ) at room temperature and with temperature (from room temperature to $473 \mathrm{~K}$ ) at a frequency of $10 \mathrm{kHz}$ are presented in Figures 8 and 9 , respectively.

The microstructure of $\mathrm{BaTiO}_{3}$ pellets (Figure 7) reveals the formation of dense ceramics with large grains of around $30 \mu \mathrm{m}$ for the BTNCs sample (Figure $7(\mathrm{a})$ ) and $\sim 100 \mu \mathrm{m}$ for BTNRs sample (Figure 7(b)). Although the particles shape of the initial hydrothermal powders was not retained, differences in the grains morphology can be observed. The BTNCs ceramics present rounded edges grains. Differently,

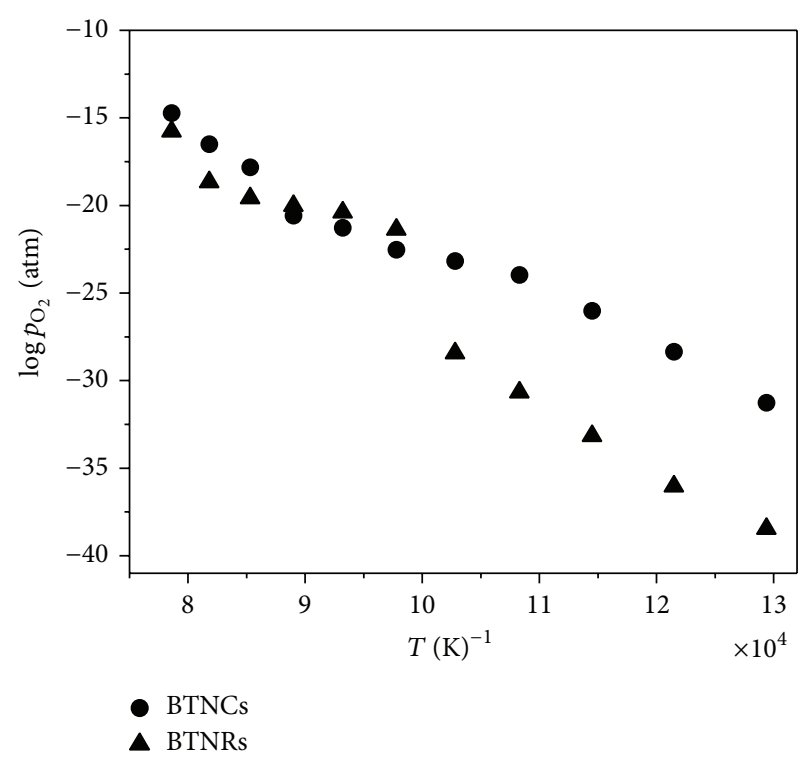

FIGURE 6: Variation of $\log p_{\mathrm{O}_{2}}$ as a function of $1 / T$ for the samples BTNCs and BTNRs.

for the BTNRs ceramics abnormally large faceted grains embedded in fine grains matrix are formed. This abnormal grain growth is well known in $\mathrm{BaTiO}_{3}$-based ceramics obtained by conventional air-sintering [45].

Figure 8(a) shows that the ceramic sample obtained with BTNRs powders exhibits two times higher dielectric permittivity at room temperature than the BTNCs ceramic. Moreover, the variation of $\varepsilon_{r}$ with the frequency is almost constant for the BTNRs and decreases with frequency increasing for the BTNCs. In Figure 8(b) one can note that for both ceramic samples the dielectric loss decreases with frequency increasing. Moreover, the values of this dielectric parameter are below 0.05 , values comparable with the earlier reports of conventionally sintered barium titanate ceramics $[46,47]$.

The first important common observation of Figures 9(a) and $9(\mathrm{~b})$ is the presence of the sharp peak in the permittivity versus temperature curves for both BTNCs and BTNRs ceramics, denoting the permittivity anomaly located at the ferroelectric to paraelectric transition temperature (Curie temperature $T_{C}$ ) [48]. The second observation is the low dielectric losses for both samples, with average values lower than 0.05 over the entire temperature range. By comparing the dielectric constant of the samples it can be observed that the BTNRs have approximately 4 times higher the maximum permittivity value than the BTNCs ceramics (at $T_{C} \varepsilon_{r}$ is 9432 for BTNRs and 2473 for BTNCs). The dielectric constant value obtained for the BTNRs sample is among the highest reported for barium titanate ceramics prepared from hydrothermal powders $[46,49,50]$. Moreover, it is known that the value of the dielectric constant of the $\mathrm{BaTiO}_{3}$ ceramics, beside the initial powders synthesis method, is also dependent on the sintering procedure and a colossal permittivity of around 40000 was reported for microwavesintered barium titanate [51]. However, the value of 9432 


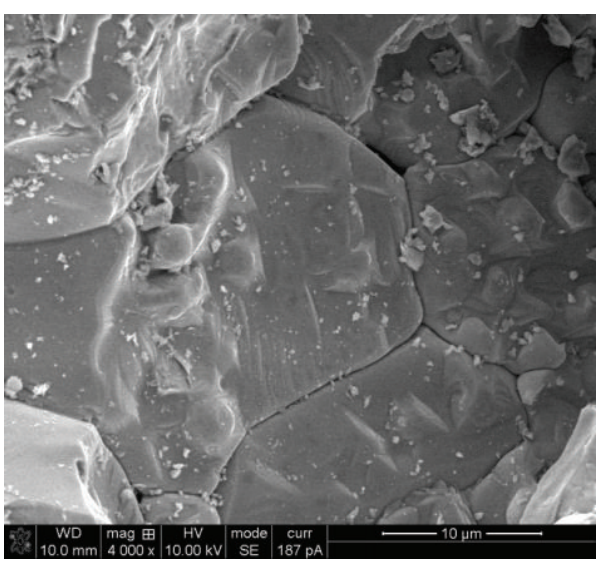

(a)

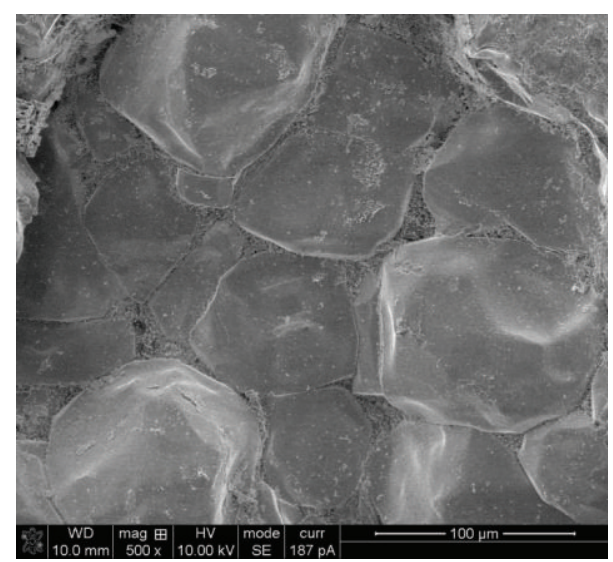

(b)

FIGURE 7: SEM microstructures of BTNCs (a) and BTNRs (b) ceramic samples.

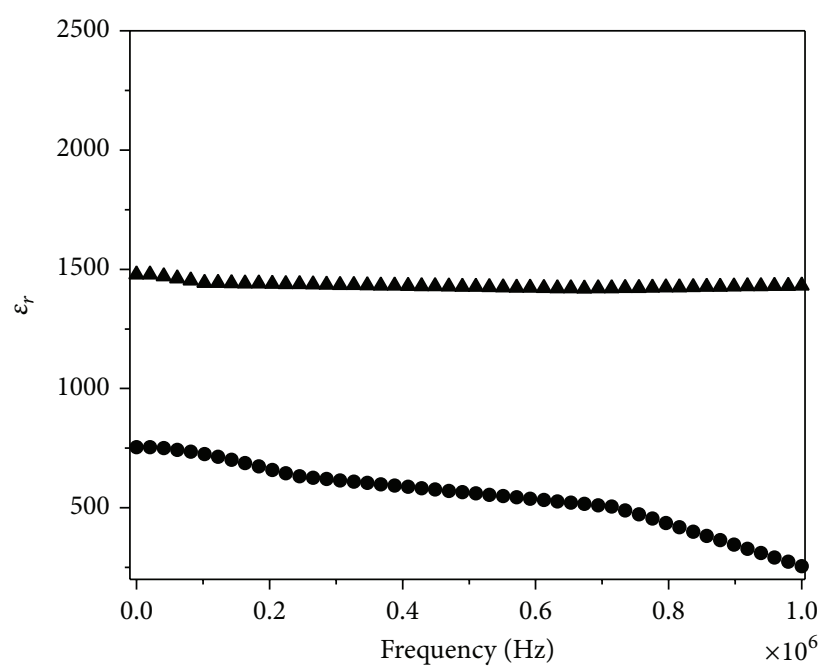

- BTNCs

- BTNRs

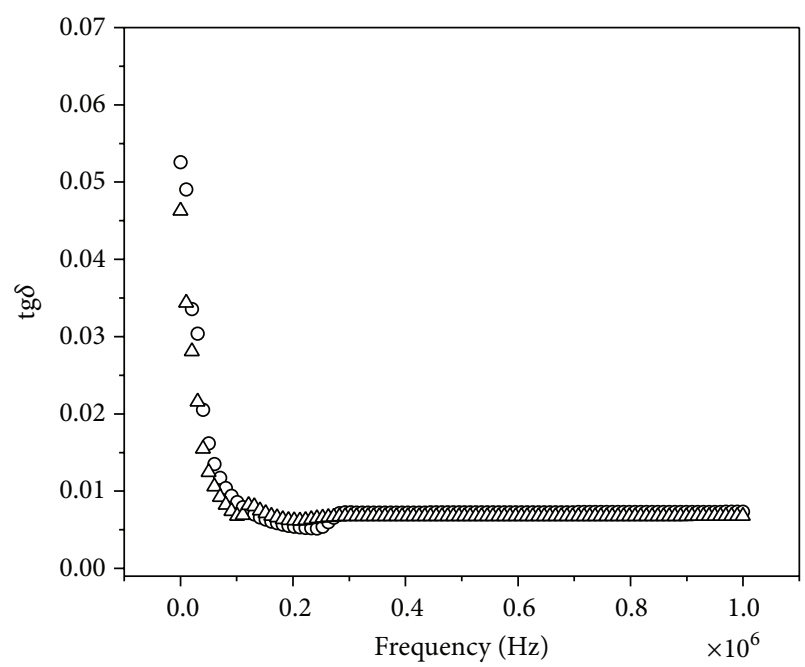

O BTNCs

$\triangle$ BTNRs

(a)

(b)

FIGURE 8: Room temperature variation of the permittivity (a) and dielectric losses (b) with frequency for BTNRs and BTNCs ceramic samples.

obtained in the present study for the BTNRs ceramics permittivity is still higher than the values normally reported for conventionally sintered barium titanate $[47,51]$. From the dependence of the permittivity on the temperature (Figure 9), the decreasing of the Curie temperature can be also noticed when hydrothermal BTNRs powders are used for the barium titanate ceramics $\left(T_{C}\right.$ is decreasing from $401 \mathrm{~K}$ for BTNCs to $396 \mathrm{~K}$ for BTNRs). Moreover the dielectric losses of BTNRs ceramics are lower than the corresponding values of BTNCs sample ( $<0.03$ for BTNCs and $<0.01$ for BTNRs). The results obtained from the dielectric properties evaluation demonstrated the enhancement of these properties when hydrothermal prepared powders with 1D morphology are used for $\mathrm{BaTiO}_{3}$ ceramics.

\section{Conclusions}

In summary, NRs and NCs of barium titanate have been effectively prepared by surfactant- and template-free hydrothermal treatment of barium acetate and titanium isopropoxide in the presence of $\mathrm{NaOH}$, at $408 \mathrm{~K}$, for $24 \mathrm{~h}$. Nanocrystals with $\sim 20 \mathrm{~nm}$ average size have been obtained. The morphological and structural investigation showed that the formation of the NRs powders, with more tetragonal structure, can be attributed to the oriented attachment of the nanocubes building blocks.

The variation of the thermodynamic properties $\Delta \bar{G}_{\mathrm{O}_{2}}$, $\Delta \bar{H}_{\mathrm{O}_{2}}$, and $\Delta \bar{S}_{\mathrm{O}_{2}}$, as well as $p_{\mathrm{O}_{2}}$ with temperature, indicated that $1 \mathrm{D} \mathrm{BaTiO}{ }_{3}$ nanopowders have the lowest concentration 


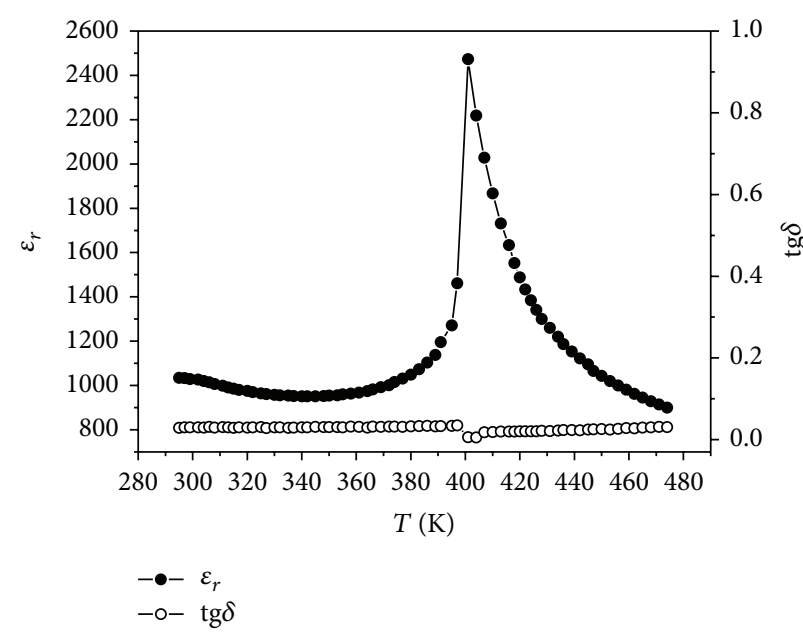

(a)

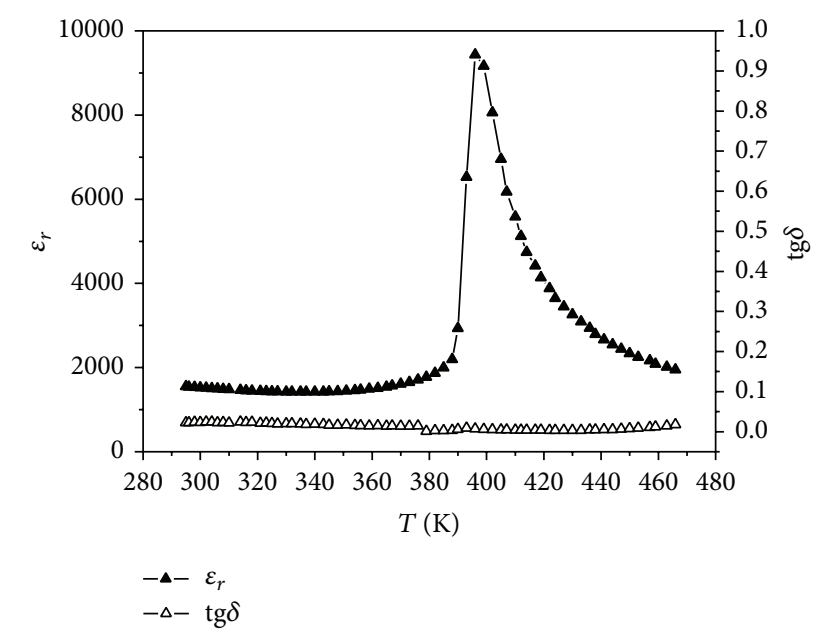

(b)

FIGURE 9: Variation of the permittivity and dielectric losses with temperature, at a $10 \mathrm{kHz}$ frequency for (a) BTNCs and (b) BTNRs ceramic samples.

of oxygen vacancies, the highest binding energy of oxygen, and the lowest concentration of oxygen vacancies randomly distributed into the perovskite lattice and the highest binding energy of oxygen.

The evaluation of the dielectric properties demonstrated that the maximum permittivity increases and the Curie point and dielectric losses decrease when NRs are used as hydrothermal nanopowders for barium titanate ceramics. These nanostructures can be promising candidates as energy harvesters in nanoelectromechanical systems (NEMS).

The results presented in this work are significant in morphology-properties correlation for the perovskite-type oxide nanomaterials, indicating the enhancement of the thermodynamic and electric properties for 1D shape.

\section{Conflict of Interests}

The authors declare that there is no conflict of interests regarding the publication of this paper.

\section{Acknowledgments}

The authors acknowledge the Romanian National Authority for Scientific Research, CNCS-UEFISCDI, for the Grant no. PN-II-RU-TE-2011-3-0160 and the Romanian Government support for the acquisition of research infrastructure under Project INFRANANOCHEM/2009-2010. Special thanks are also addressed to Manuela Tanase for experimental help and to Dr. Irina Atkinson and Dr. Simona Petrescu for XRD and Raman measurements, respectively. Many acknowledgments are addressed to the members of the Electroceramics group of Prof. Dr. Paula Vilarinho, from CICECO University of Aveiro, Portugal, for assistance regarding the experimental procedure for the electric properties evaluation.

\section{References}

[1] N. Izyumskaya, Y. Alivov, and H. Morkoç, "Oxides, oxides, and more oxides: high-oxides, ferroelectrics, ferromagnetics, and multiferroics," Critical Reviews in Solid State and Materials Sciences, vol. 34, no. 3-4, pp. 89-179, 2009.

[2] M. M. Vijatovic, J. D. Bobic, and B. D. Stojanovic, "History and challenges of barium titanate. Part II," Science of Sintering, vol. 40 , no. 3, pp. 235-244, 2008.

[3] J. Rödel, W. Jo, K. T. P. Seifert, E.-M. Anton, T. Granzow, and D. Damjanovic, "Perspective on the development of lead-free piezoceramics," Journal of the American Ceramic Society, vol. 92, no. 6, pp. 1153-1177, 2009.

[4] G. Arlt, D. Hennings, and G. Dewith, "Dielectric properties of fine-grained barium titanate ceramics," Journal of Applied Physics, vol. 58, no. 4, pp. 1619-1625, 1985.

[5] A. N. Morozovska, E. A. Eliseev, and M. D. Glinchuk, "Ferroelectricity enhancement in confined nanorods: direct variational method," Physical Review B-Condensed Matter and Materials Physics, vol. 73, no. 21, Article ID 214106, 2006.

[6] S. Y. Zhang, F. S. Jiang, G. Qu, and C. Y. Lin, "Synthesis of single-crystalline perovskite barium titanate nanorods by a combined route based on sol-gel and surfactant-templated methods," Materials Letters, vol. 62, no. 15, pp. 2225-2228, 2008.

[7] B. A. Hernandez, K.-S. Chang, E. R. Fisher, and P. K. Dorhout, "Sol-gel template synthesis and characterization of $\mathrm{BaTiO}_{3}$ and $\mathrm{PbTiO}_{3}$ nanotubes," Chemistry of Materials, vol. 14, no. 2, pp. 480-482, 2002.

[8] S. J. Limmer and G. Cao, "Sol-gel electrophoretic deposition for the growth of oxide nanorods," Advanced Materials, vol. 15, no. 5, pp. 427-431, 2003.

[9] K. Žagar, F. Hernandez-Ramirez, J. D. Prades, J. R. Morante, A. Rečnik, and M. Čeh, "Characterization of individual barium titanate nanorods and their assessment as building blocks of new circuit architectures," Nanotechnology, vol. 22, no. 38, Article ID 385501, 2011.

[10] A. Koka, Z. Zhou, H. Tang, and H. A. Sodano, "Controlled synthesis of ultra-long vertically aligned $\mathrm{BaTiO}_{3}$ nanowire 
arrays for sensing and energy harvesting applications," Nanotechnology, vol. 25, no. 37, Article ID 375603, 2014.

[11] A. Lamberti, N. Garino, K. Bejtka et al., "Synthesis of ferroelectric $\mathrm{BaTiO}_{3}$ tube-like arrays by hydrothermal conversion of a vertically aligned $\mathrm{TiO}_{2}$ nanotube carpet," New Journal of Chemistry, vol. 38, no. 5, pp. 2024-2030, 2014.

[12] N. P. Padture and X. Wei, "Hydrothermal synthesis of thin films of barium titanate ceramic nano-tubes at $200^{\circ} \mathrm{C}$," Journal of the American Ceramic Society, vol. 86, no. 12, pp. 2215-2217, 2003.

[13] F. Maxim, Bottom up strategies for the morphology control of $\mathrm{BaTiO}_{3}$ particulates [Ph.D. thesis], University of Aveiro, Aveiro, Portugal, 2010.

[14] N. Bao, L. Shen, G. Srinivasan, K. Yanagisawa, and A. Gupta, "Shape-controlled monocrystalline ferroelectric barium titanate nanostructures: from nanotubes and nanowires to ordered nanostructures," The Journal of Physical Chemistry C, vol. 112, no. 23, pp. 8634-8642, 2008.

[15] Y. Cao, K. Zhu, Q. Wu, Q. Gu, and J. Qiu, "Hydrothermally synthesized barium titanate nanostructures from $\mathrm{K}_{2} \mathrm{Ti}_{4} \mathrm{O}_{9}$ precursors: morphology evolution and its growth mechanism," Materials Research Bulletin, vol. 57, pp. 162-169, 2014.

[16] F. Maxim, P. Ferreira, and P. Vilarinho, "Strategies for the structure and morphology control of $\mathrm{BaTiO}_{3}$ nanoparticles," in New Applications of Nanomaterials Micro and Nanoengineering, A. C. Ion, D. Dascalu, G. Carja, and M. L. Ciurea, Eds., pp. 8397, Editura Academiei Romane, Bucharest, Romania, 2014.

[17] Y. Xia, P. Yang, Y. Sun et al., "One-dimensional nanostructures: Synthesis, characterization, and applications," Advanced Materials, vol. 15, no. 5, pp. 353-389, 2003.

[18] A. Magrez, E. Vasco, J. W. Seo, C. Dicker, N. Setter, and L. Forró, "Growth of single-crystalline $\mathrm{KNbO}_{3}$ nanostructures," The Journal of Physical Chemistry B, vol. 110, no. 1, pp. 58-61, 2006.

[19] E. Vasco, A. Magrez, L. Forró, and N. Setter, "Growth kinetics of one-dimensional $\mathrm{KNbO}_{3}$ nanostructures by hydrothermal processing routes," The Journal of Physical Chemistry B, vol. 109, no. 30, pp. 14331-14334, 2005.

[20] F. Maxim, P. Ferreira, and P. M. Vilarinho, "Morphology control of $\mathrm{BaTiO}_{3}$ nanoparticles prepared by sol-gel hydrothermal method," Microscopy and Microanalysis, vol. 18, no. 5, pp. 111112, 2012.

[21] S. Tanasescu, N. D. Totir, and D. I. Marchidan, "Thermodynamic data of the perovskite-type $\mathrm{LaMnO}_{3 \pm x}$ and $\mathrm{La}_{0.7} \mathrm{Sr}_{0.3} \mathrm{MnO}_{3 \pm x}$ by a solid-state electrochemical technique," Electrochimica Acta, vol. 43, no. 12-13, pp. 1675-1681, 1998.

[22] C. J. Murphy and N. R. Jana, "Controlling the aspect ratio of inorganic nanorods and nanowires," Advanced Materials, vol. 14, no. 1, pp. 80-82, 2002.

[23] R. L. Penn and J. F. Banfield, “Oriented attachment and growth, twinning, polytypism, and formation of metastable phases: insights from nanocrystalline $\mathrm{TiO}_{2}$," The American Mineralogist, vol. 83, pp. 1077-1082, 1998.

[24] J. Zhang, F. Huang, and Z. Lin, "Progress of nanocrystalline growth kinetics based on oriented attachment," Nanoscale, vol. 2, no. 1, pp. 18-34, 2010.

[25] R. L. Penn and J. F. Banfield, "Imperfect oriented attachment: dislocation generation in defect-free nanocrystals," Science, vol. 281, no. 5379, pp. 969-971, 1998.

[26] D. Hennings and S. Schreinemacher, "Characterization of hydrothermal barium titanate," Journal of the European Ceramic Society, vol. 9, no. 1, pp. 41-46, 1992.
[27] E.-W. Shi, C.-T. Xia, W.-Z. Zhong, B.-G. Wang, and C.-D. Feng, "Crystallographic properties of hydrothermal barium titanate crystallites," Journal of the American Ceramic Society, vol. 80, no. 6, pp. 1567-1572, 1997.

[28] D. F. K. Hennings, C. Metzmacher, and B. S. Schreinemacher, "Defect chemistry and microstructure of hydrothermal barium titanate," Journal of the American Ceramic Society, vol. 84, no. 1, pp. 179-182, 2001.

[29] M. Vijatovic, J. Bobic, and B. Stojanovic, "History and challenges of barium titanate: part I," Science of Sintering, vol. 40, no. 2, pp. 155-165, 2008.

[30] T. Noma, S. Wada, M. Yano, and T. Suzuki, "Analysis of lattice vibration in fine particles of barium titanate single crystal including the lattice hydroxyl group," Journal of Applied Physics, vol. 80, no. 9, pp. 5223-5233, 1996.

[31] Y. Shiratori, C. Pithan, J. Dornseiffer, and R. Waser, "Raman scattering studies on nanocrystalline $\mathrm{BaTiO}_{3}$ Part I-isolated particles and aggregates," Journal of Raman Spectroscopy, vol. 38, no. 10, pp. 1288-1299, 2007.

[32] R. J. Li, W. X. Wei, J. L. Hai, L. X. Gao, Z. W. Gao, and Y. Y. Fan, "Preparation and electric-field response of novel tetragonal barium titanate," Journal of Alloys and Compounds, vol. 574, pp. 212-216, 2013.

[33] P. R. Ren, H. Q. Fan, X. Wang, and K. Liu, "A novel approach to prepare tetragonal $\mathrm{BaTiO}_{3}$ nanopowders," Materials Letters, vol. 65, no. 2, pp. 212-214, 2011.

[34] J. Q. Qi, T. Peng, Y. M. Hu et al., "Direct synthesis of ultrafine tetragonal $\mathrm{BaTiO}_{3}$ nanoparticles at room temperature," Nanoscale Research Letters, vol. 6, article 466, pp. 1-4, 2011.

[35] Y. Xie, S. Yin, T. Hashimoto, Y. Tokano, A. Sasaki, and T. Sato, "Low temperature synthesis of tetragonal $\mathrm{BaTiO}_{3}$ by a novel composite-hydroxide-mediated approach and its dielectric properties," Journal of the European Ceramic Society, vol. 30, no. 3, pp. 699-704, 2010.

[36] I. Tsuyumoto, M. Kobayashi, T. Are, and N. Yamazaki, "Nanosized tetragonal $\mathrm{BaTiO}_{3}$ powders synthesized by a new peroxoprecursor decomposition method," Chemistry of Materials, vol. 22, no. 9, pp. 3015-3020, 2010.

[37] R. Vivekanandan and T. R. N. Kutty, "Characterization of barium titanate fine powders formed from hydrothermal crystallization," Powder Technology, vol. 57, no. 3, pp. 181-192, 1989.

[38] U. Robels and G. Arlt, "Domain wall clamping in ferroelectrics by orientation of defects," Journal of Applied Physics, vol. 73, no. 7, pp. 3454-3460, 1993.

[39] G. Arlt and H. Neumann, "Internal bias in ferroelectric ceramics: origin and time dependence," Ferroelectrics, vol. 87, no. 1, pp. 109-120, 1988.

[40] C.-R. Song and H.-I. Yoo, "Chemical diffusivity of $\mathrm{BaTiO}_{3-\delta}$ III. Conductivity-nonstoichiometry $(\delta)$ correlation in a mixed $\mathrm{n} / \mathrm{p}$ regime," Solid State Ionics, vol. 124, no. 3, pp. 289-299, 1999.

[41] P. Erhart and K. Albe, "Thermodynamics of mono-and divacancies in barium titanate," Journal of Applied Physics, vol. 102, no. 8, Article ID 084111, 2007.

[42] S. Tanasescu, Z. Yáng, J. Martynczuk et al., "Effects of A-site composition and oxygen nonstoichiometry on the thermodynamic stability of compounds in the Ba-Sr-Co-Fe-O system," Journal of Solid State Chemistry, vol. 200, pp. 354-362, 2013.

[43] D.-K. Lee and H.-I. Yoo, "Oxygen nonstoichiometry of undoped $\mathrm{BaTiO}_{3-\delta}$, Solid State Ionics, vol. 144, no. 1-2, pp. 87-97, 2001.

[44] H.-S. Kwon, H.-I. Yoo, C.-H. Kim, and K.-H. Hur, "Semiconductor-to-insulator transition of undoped-BaTiO 
in quenched state," Journal of Applied Physics, vol. 107, no. 8, Article ID 083702, 2010.

[45] B.-K. Lee, S.-Y. Chung, and S.-J. L. Kang, "Grain boundary faceting and abnormal grain growth in $\mathrm{BaTiO}_{3}$," Acta Materialia, vol. 48, no. 7, pp. 1575-1580, 2000.

[46] P. Pinceloup, C. Courtois, A. Leriche, and B. Thierry, "Hydrothermal synthesis of nanometer-sized barium titanate powders: control of barium/titanium ratio, sintering, and dielectric properties," Journal of the American Ceramic Society, vol. 82, no. 11, pp. 3049-3056, 1999.

[47] J.-C. M’Peko, J. S. C. Francis, and R. Raj, "Field-assisted sintering of undoped $\mathrm{BaTiO}_{3}$ : microstructure evolution and dielectric permittivity," Journal of the European Ceramic Society, vol. 34, pp. 3655-3660, 2014.

[48] D. Hennings, M. Klee, and R. Waser, "Advanced dielectrics: bulk ceramics and thin Films," Advanced Materials, vol. 3, no. 7-8, pp. 334-340, 1991.

[49] P. K. Dutta, R. Asiaie, S. A. Akbar, and W. D. Zhu, "Hydrothermal synthesis and dielectric properties of tetragonal $\mathrm{BaTiO}_{3}$," Chemistry of Materials, vol. 6, no. 9, pp. 1542-1548, 1994.

[50] N. J. Joshi, G. S. Grewal, V. Shrinet, T. P. Govindan, and A. Pratap, "Synthesis and dielectric behavior of nano-scale barium titanate," IEEE Transactions on Dielectrics and Electrical Insulation, vol. 19, no. 1, pp. 83-90, 2012.

[51] H. Han, D. Ghosh, J. L. Jones, and J. C. Nino, "Colossal permittivity in microwave-sintered barium titanate and effect of annealing on dielectric properties," Journal of the American Ceramic Society, vol. 96, no. 2, pp. 485-490, 2013. 

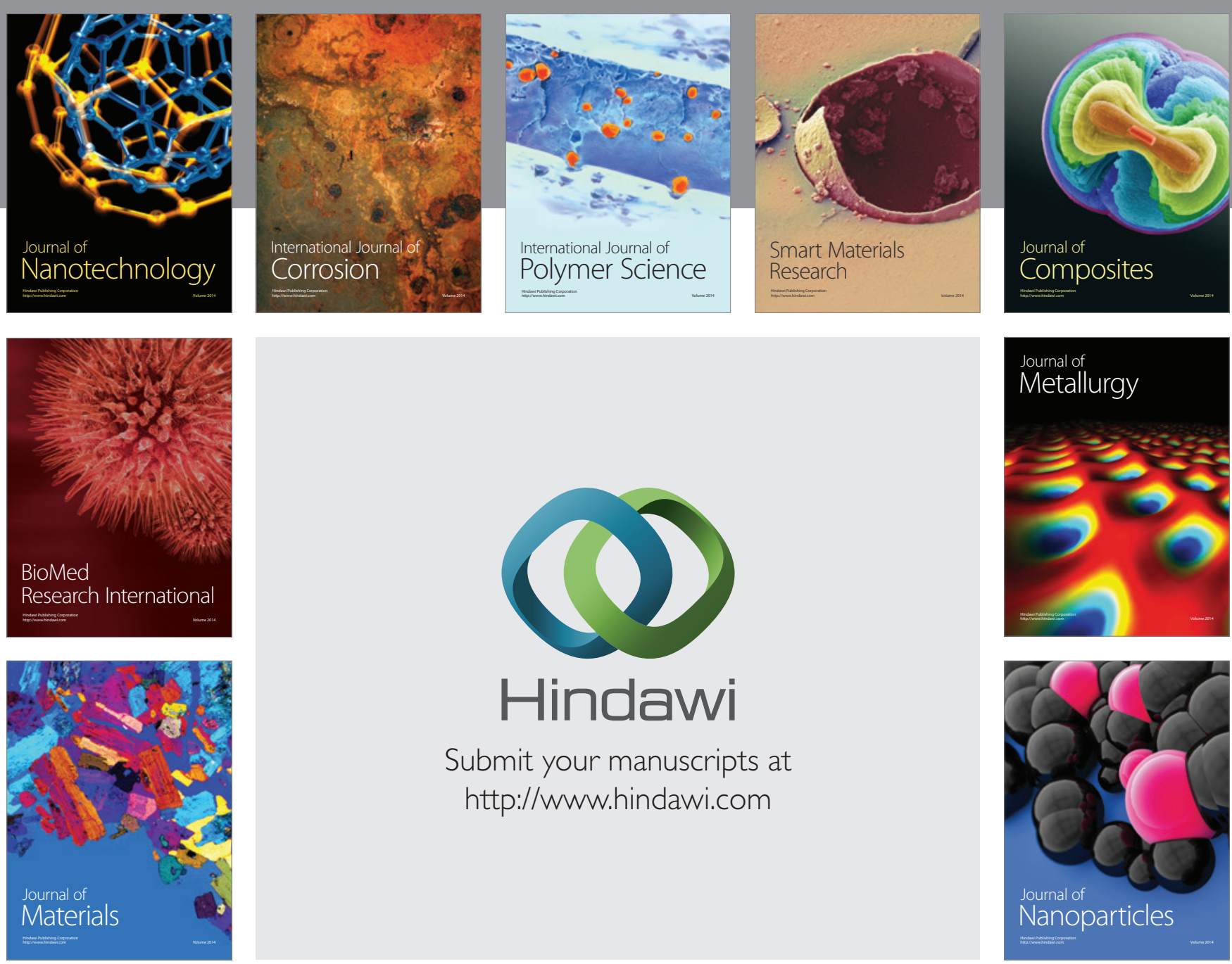

Submit your manuscripts at http://www.hindawi.com
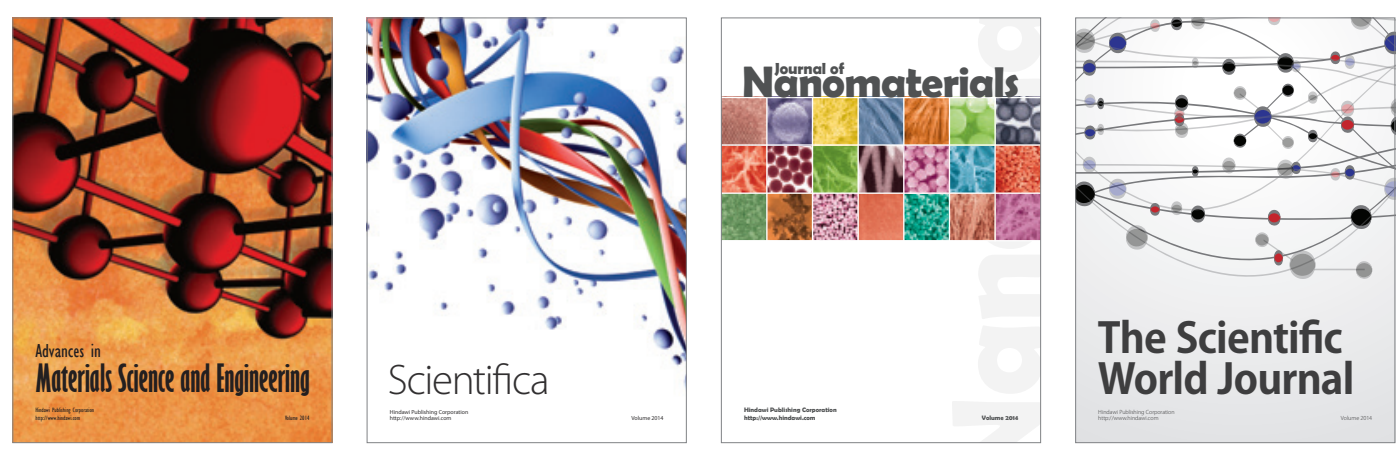

\section{The Scientific World Journal}
